\title{
Direct differentiation of atrial and ventricular myocytes from human embryonic stem cells by alternating retinoid signals
}

Qiangzhe Zhang ${ }^{1, *}$, Junjie Jiang ${ }^{1, *}$, Pengcheng Han ${ }^{1, *}$, Qi Yuan ${ }^{1}$, Jing Zhang ${ }^{1}$, Xiaoqian Zhang ${ }^{1}$, Yanyan Xu ${ }^{1}$, Henghua Cao ${ }^{1}$, Qingzhang Meng ${ }^{1}$, Li Chen ${ }^{1}$, Tian Tian ${ }^{1}$, Xin Wang ${ }^{2}, \mathrm{Pu} \mathrm{Li}^{1}$, Jurgen Hescheler ${ }^{3}$, Guangju Ji ${ }^{1}$, Yue $\mathrm{Ma}^{1}$

${ }^{I}$ National Laboratory of Biomacromolecules, Institute of Biophysics, Chinese Academy of Sciences, 15 Datun Rd., Chaoyang District, Beijing 100101, China; ${ }^{2}$ Institute of Biochemistry and Cell Biology, Shanghai Institutes of Biological Sciences, 320 Yueyang Rd., Shanghai 200032, China; ${ }^{3}$ Center for Physiology and Pathophysiology, Institute for Neurophysiology, University of Cologne, Robert-Koch-Str. 39, 50931 Cologne, Germany

Although myocyte cell transplantation studies have suggested a promising therapeutic potential for myocardial infarction, a major obstacle to the development of clinical therapies for myocardial repair is the difficulties associated with obtaining relatively homogeneous ventricular myocytes for transplantation. Human embryonic stem cells (hESCs) are a promising source of cardiomyocytes. Here we report that retinoid signaling regulates the fate specification of atrial versus ventricular myocytes during cardiac differentiation of hESCs. We found that both Noggin and the panretinoic acid receptor antagonist BMS-189453 (RAi) significantly increased the cardiac differentiation efficiency of hESCs. To investigate retinoid functions, we compared Noggin+RAi-treated cultures with Noggin+RA-treated cultures. Our results showed that the expression levels of the ventricular-specific gene $I R X-4$ were radically elevated in Noggin+RAi-treated cultures. MLC-2V, another ventricular-specific marker, was expressed in the majority of the cardiomyocytes in Noggin+RAi-treated cultures, but not in the cardiomyocytes of Noggin+RA-treated cultures. Flow cytometry analysis and electrophysiological studies indicated that with $64.7 \pm 0.88 \%$ (mean \pm s.e.m) cardiac differentiation efficiency, $83 \%$ of the cardiomyocytes in Noggin+RAi-treated cultures had embryonic ventricular-like action potentials (APs). With $50.7 \pm \mathbf{1 . 7 6} \%$ cardiac differentiation efficiency, $\mathbf{9 4 \%}$ of the cardiomyocytes in Noggin+RAtreated cultures had embryonic atrial-like APs. These results were further confirmed by imaging studies that assessed the patterns and properties of the $\mathrm{Ca}^{2+}$ sparks of the cardiomyocytes from the two cultures. These findings demonstrate that retinoid signaling specifies the atrial versus ventricular differentiation of hESCs. This study also shows that relatively homogeneous embryonic atrial- and ventricular-like myocyte populations can be efficiently derived from hESCs by specifically regulating Noggin and retinoid signals.

Keywords: human embryonic stem cell; cardiac subtype specification; heart development

Cell Research (2011) 21:579-587. doi:10.1038/cr.2010.163; published online 23 November 2010

\section{Introduction}

Using current technologies, embryonic atrial-, ventric-

\footnotetext{
*These three authors contributed equally to this work.

Correspondence: Yue $\mathrm{Ma}^{\mathrm{a}}$, Guangju Ji

a'E-mail: yuema@ibp.ac.cn

bE-mail: gj28@ibp.ac.cn

Received 30 September 2010; revised 3 November 2010; accepted 8 November 2010; published online 23 November 2010
}

ular- and nodal-like cardiomyocytes can be differentiated nonspecifically from hESCs [1-4]. Swine transplantation studies have shown that implanted hESC-derived cardiomyocytes have pace-making activities, which are a potential cause of ventricular arrhythmias [5]. The application of hESCs in myocardial repair is hampered by this cardiac subtype heterogeneity of hESC-derived cardiomyocytes [6]. To direct the differentiation of hESCs into a desired cardiac subtype, the mechanisms of cardiac subtype specification must be uncovered. Although sev- 
eral growth factors, such as activin A, bone morphogenetic protein 4 (BMP4), wnt-3a, basic fibroblast growth factor (bFGF) and dickkopf homolog 1 (DKK1), promote cardiogenesis and are used in several hESC cardiac differentiation protocols $[2,3,7]$, there is no evidence to date showing that these or other growth factors regulate cardiac subtype specification during hESC differentiation. Identifying the key regulators of cardiac subtype specification is critical for reducing the heterogeneity of the hESC-derived cardiomyocyte population. Reducing the heterogeneity of this population will be important for its later use in regenerative medicine or in drug testing systems $[1,8]$.

BMP signaling is tightly controlled during mesoderm and heart development. In mouse embryos, the BMP antagonist Noggin is transiently but strongly expressed in the cardiac crescent at embryonic day E7.5 to E8.0 [9]. Dkk1, a potent inducer of heart development [3, 10, 11], synergises with BMP antagonism to specify heart tissue in non-cardiogenic mesoderm from Xenopus embryos [12]. It has been shown that long-term treatment of hESCs with BMP4 induces trophoblast-like cell differentiation [13], while short-term treatment initiates mesoderm formation [14]. Together, these results suggest that inhibiting BMP signaling after mesoderm formation facilitates cardiac development.

RA signaling restricts the cardiac progenitor pool, and exposure of the anterior lateral plate mesoderm of zebrafish embryos to the RA antagonist BMS-189453 causes uncommitted lateral mesodermal cells to become myocardial progenitors $[15,16]$. RA signaling also regulates anterior-posterior polarisation of the heart [17]. Chicken transplantation studies have revealed that the cardiogenic mesoderm from $\mathrm{HH}$ stages 4-6, originally fated to be atria, is competent to develop into functional ventricles, and vice versa $[18,19]$. RA treatment of $\mathrm{HH}$ stage 4 cardiogenic tissue activates the expression of the atrium-specific gene $A M H C 1$ in anterior progenitors fated to develop into out-flow track tissues [20]. Furthermore, in both mouse and chicken embryos, inhibition of RA signaling within critical periods produces embryos with oversized ventricles and smaller or missing atria, and the exogenous addition of RA results in reverted phenotypes $[21,22]$. Furthermore, studies with mouse embryonic stem cells indicate that retinoic acid promotes the expression of atrial-related genes [23].

Based on these previous studies, we hypothesised that inhibition of the BMP pathway after the initiation of hESC differentiation and blocking retinoic acid signaling would promote cardiogenesis. Retinoid signaling may also regulate the atrial versus ventricular differentiation of hESCs. To test these hypotheses, we added Noggin,
RA and the RA inhibitor RAi to cardiac differentiation cultures at different time intervals and investigated the effects of these factors on the cardiogenesis and cardiac subtype specification of hESC derivatives. Our results indicate that the inhibition of both BMP and RA signals with Noggin and RAi significantly promotes cardiogenesis, and retinoid signaling controls the atrial versus ventricular specification of differentiated hESCs. In addition to providing important insights into the mechanisms that specify cardiac subtypes, our findings also demonstrate the direct differentiation of relatively homogeneous embryonic atrial- and ventricular-like myocytes from hESCs.

\section{Results}

Noggin and the RA antagonist BMS-189453 promote the cardiogenesis of differentiated hESCs

To investigate its functions in cardiac differentiation, Noggin was systematically added at different time intervals between days 2 and 5 to cardiomyocyte-differentiating $\mathrm{hESC}$ cultures generated by a protocol developed in our laboratory (see Methods for a detailed description). The results show that cardiac differentiation was slightly repressed when Noggin was present between days 2 and 3 , while differentiation was significantly promoted when Noggin was present between days 2.5 and 4.5. The highest cardiac differentiation efficiency was achieved when Noggin was applied between days 4 and 5 (Figure 1B). Western blotting for phosphorylated Smad 1, 5 and 8 indicated that Noggin reduced the downstream activities of BMP signaling (data not shown). Therefore, the inhibition of BMP signaling promotes cardiogenesis in hESCs after the initiation of differentiation.

Previous reports that RA signaling restricts the embryonic cardiac progenitor pool raise the possibility that inhibition of RA signaling during cardiac differentiation of hESCs could promote cardiogenesis. Vitamin A, the substrate for RA synthesis, and RALDH2, the enzyme responsible for RA synthesis [21], were both present in our cultures (data not shown), suggesting that RA signaling was potentially activated. Therefore, we tested the effects of RA inhibition on hESC cardiac differentiation by adding RAi to our cardiomyocyte-differentiating cultures between days 4 and 9 (Figure 1C). Flow cytometry analysis indicated that cardiac differentiation efficiencies were markedly increased when RAi was added between days 6 and 9 (Figure. 1C). These results demonstrated that inhibition of RA signaling promotes cardiac differentiation of hESCs.

Next, we combined Noggin treatment on days 4 and 5 with RAi treatment on days 6,7 and 8 . Flow cytometry 
analysis of $\mathrm{CTNT}^{+}$cells from day 14 cultures showed that the differentiation efficiency with Noggin alone was $50 \pm 3.06 \%$ (mean \pm s.e.m.), and this efficiency increased to $73 \pm 2.08 \%$ when the cells were treated with both RAi and Noggin (Figure 1D). This result was confirmed by quantitative RT-PCR analysis of day 14 cultures. The expression levels of both CTNT and NKX2.5 were significantly higher in the Noggin+RAi-treated cultures than in cultures treated with Noggin alone (Figure 1E). Immunostaining indicated the expression of typical cardiac markers, including CTNT, $\alpha$-Actinin, MLC-2A, MLC$2 \mathrm{~V}$, and $\beta-\mathrm{MHC}$, in these cultured cells (Figure $1 \mathrm{~F}$ ).

Alternative retinoid signals direct the differentiation of hESCs into two distinct subtypes of cardiomyocytes
Chicken and mouse embryo studies indicated that retinoid signaling regulates the fate specification of inflow and out-flow track tissues [17-22]. Therefore, we hypothesized that the activation or inactivation of retinoid signaling directs the atrial vs. ventricular fate specification of differentiated hESC cardiac progenitors, and such a mechanism could be used to efficiently generate either hESC-derived atrial- or ventricular-like myocytes.

To test this hypothesis, either RA or its antagonist, RAi, was added to the Noggin-treated cultures between days 6 and 8 in parallel experiments (Figure 1A). After 14 days of differentiation, the frequencies of $\mathrm{CTNT}^{+}$cells in the Noggin+RA- and Noggin+RAi-treated cultures were $50.7 \pm 1.76 \%$ and $64.7 \pm 0.88 \%$, respectively (Figure $2 \mathrm{~A}$ ). While there was only a $14 \%$ difference in the

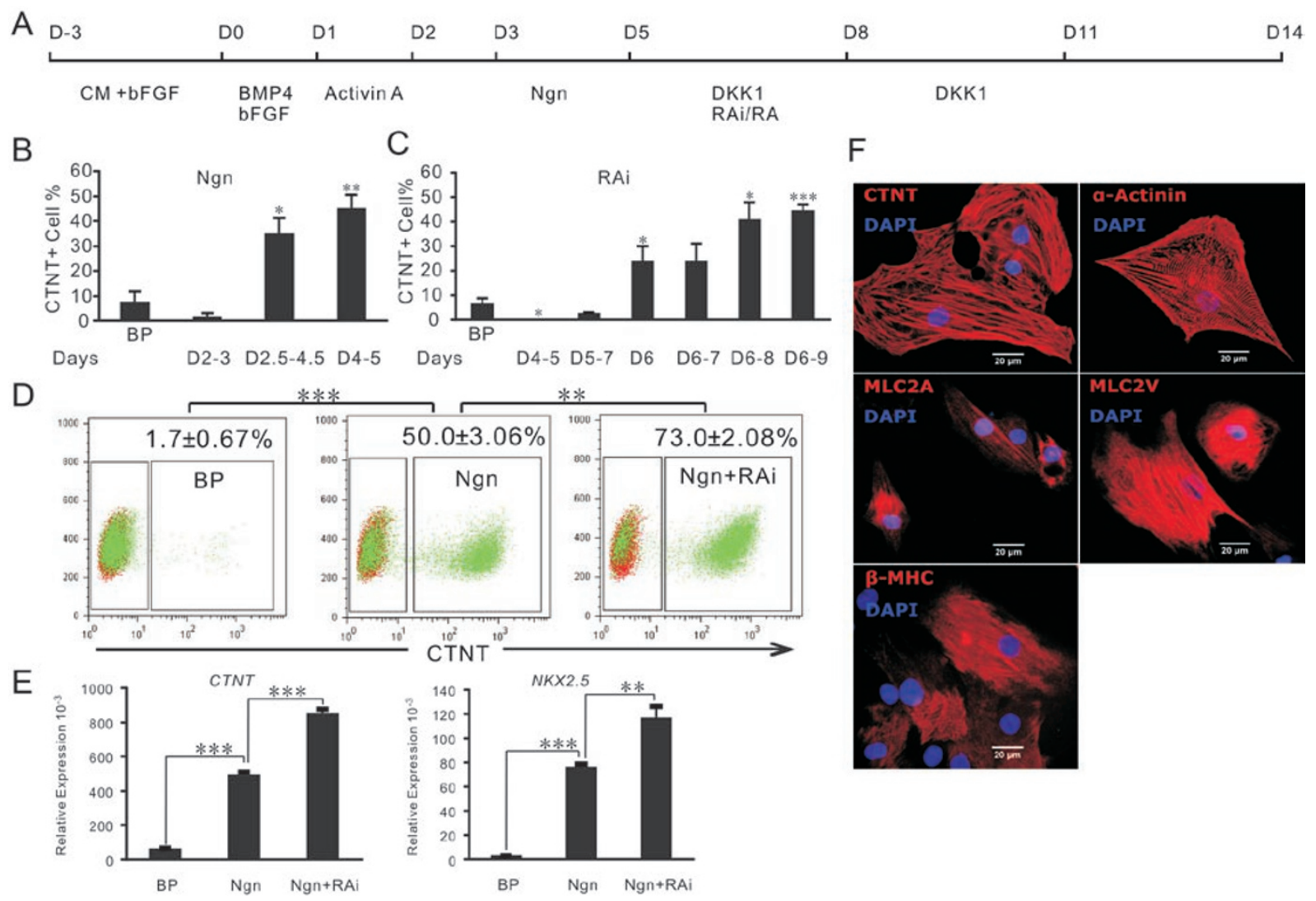

Figure 1 Noggin (Ngn) and RA inhibition promote hESC cardiac differentiation. (A) An outline of the protocol used for the differentiation of hESCs to cardiac lineages. (B) Frequencies of CTNT $^{+}$cells at day 14; cardiac-induced cultures with addition of $\mathrm{Ngn}$ at the time intervals indicated; the basic protocol (BP) was used as a control. (C) Frequencies of $\mathrm{CTNT}^{+}$cells in day 14 cardiac-induced cultures with addition of the RA inhibitor, RAi, at the indicated time intervals; the BP was used as a control. (D) Flow cytometry analysis of $\mathrm{CTNT}^{+}$cells in cultures with the BP used as a control; BP cultures with Ngn added at days 4 and 5, and BP cultures with Ngn added at days 4 and 5 and RAi added from day 6 to day 8 (Ngn+RAi). (E) Quantitative RTPCR gene expression analysis on day 14 BP, Ngn-treated, and Ngn+RAi-treated cultures, as indicated in (D). The average expression, normalized to GADPH, is shown. (F) Immunostaining analysis of cardiac-induced cultures. Where shown, bars represent the standard error of the mean of three independent experiments. $\mathrm{CM}$, conditioned medium. $P$ compared with the BP control or otherwise indicated: $* P<0.05, * * P<0.005, * * * P<0.0005$. 
differentiation efficiencies, the size of the beating cardiomyocytes in the Noggin+RA-treated cultures was smaller than that of those in the Noggin+RAi-treated cultures (Figure 2B and 2D). The beating rate of the cardiomyocytes in the Noggin+RA-treated cultures was also faster than that of those in the Noggin+RAi-treated cultures (Figure 2C and Supplementary information, Movies S1, S2), suggesting that there were two different subtypes of cardiomyocytes present in these two different cultures.

Next, we examined the expression of ventricular-

A

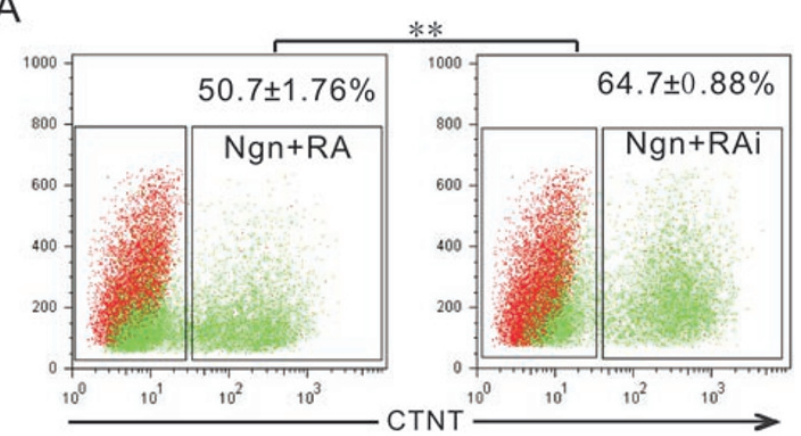

B

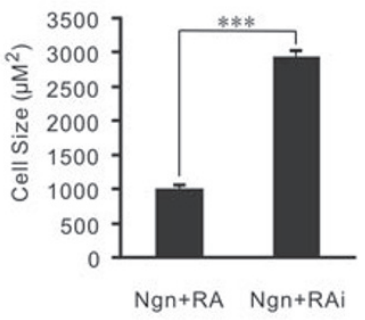

$\mathrm{C}$

\section{$\mathrm{D}$}
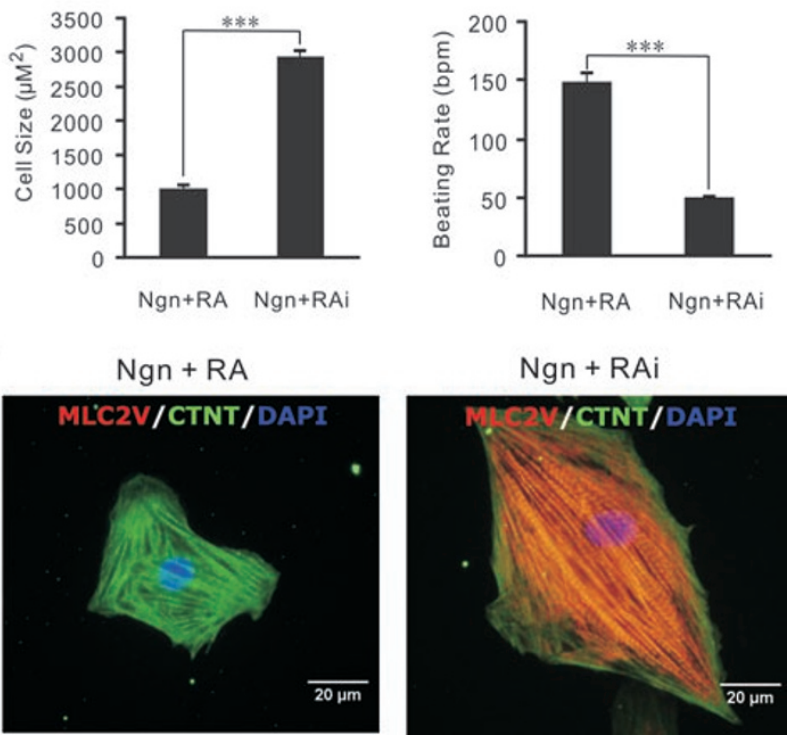

Figure 2 Morphologies and beating rates of differentiated cardiomyocytes. (A) Flow cytometry analysis of $\mathrm{CTNT}^{+}$cells from day 14 cultures differentiated with the BP plus treatments with Ngn at days 4 and 5 , and RA (Ngn+RA) or RAi (Ngn+RAi.) treatment from days 6 to 8 . (B) The size of single cardiomyocytes from 60-day-old cultures treated with $\mathrm{Ngn}+\mathrm{RA}(n=35)$ and $\mathrm{Ngn}+\mathrm{RAi}$ $(n=31)$, measured with Image J software. (C) The beating rates (beat/minute) of 60-day-old differentiated cultures treated with $\mathrm{Ngn}+\mathrm{RA}$ or Ngn+RAi $(n=4)$. (D) Immunostaining of single cells from 60-day-old differentiated cultures treated with $\mathrm{Ngn}+\mathrm{RA}$ or Ngn+RAi. $* * P<0.005,{ }^{* * *} P<0.0005$.
A

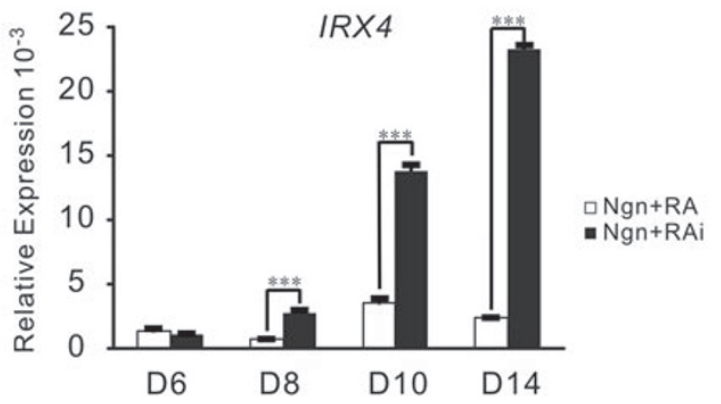

B
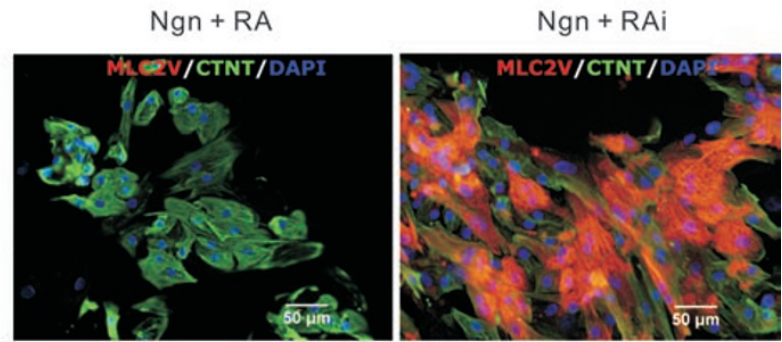

C

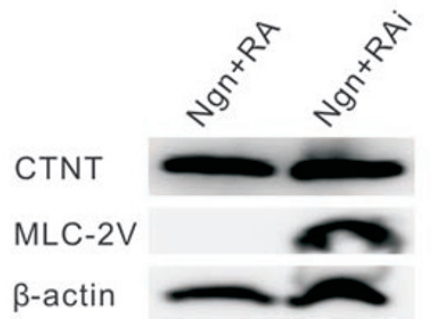

Figure 3 Characterization of hESC-derived cardiomyocytes induced by Ngn+RA and Ngn+RAi. (A) Quantitative RT-PCR analysis of the kinetics of $I R X 4$ gene expression in Ngn+RA and $\mathrm{Ngn}+\mathrm{RAi}$ treated cultures. The average expression, normalized to GAPDH, is shown. (B) Immunostaining of 60-day-old Ngn+RA- and Ngn+RAi-induced cultures demonstrated MLC$2 \mathrm{~V}$ expression in the majority of $\mathrm{CTNT}^{+}$cells of Ngn+RAi-treated cultures but not in those of Ngn+RA-treated cultures. (C) Western blotting of 60-day-old Ngn+RA- and Ngn+RAi-treated cultures indicated that even though CTNT is evenly expressed in both cultures, MLC-2V is strongly expressed in Ngn+RAi-treated cultures, but not in Ngn+RA-treated cultures. ${ }^{* * *} P<0.0005$.

specific genes (IRX4 and $M L C-2 V)$ in the two cultures $[22,24,25]$. Quantitative RT-PCR showed that in the Noggin+RAi-treated cultures, IRX4 expression started to rise at day 8 , and it was 10 -fold greater by day 14 than the Noggin+RA-treated cultures (Figure 3A). Immunostaining of 60-day-old cultures showed that MLC$2 \mathrm{~V}$ was expressed in the majority of $\mathrm{CTNT}^{+}$cells in the Noggin+RAi-treated cultures, but not in the Noggin+RAtreated cultures (Figure 3B). This result is consistent with the results from western blotting experiments that 
indicated that although CTNT was expressed at similar levels in these two cultures, MLC-2V was strongly and only expressed in the Noggin+RAi-treated cultures (Figure $3 \mathrm{C}$ ).

We also compared the expression levels of CTNT and MLC-2V in Noggin+RAi-, Noggin- and Noggin+RAtreated cultures by immunostaining and western blots. The results show that in the Noggin-only-treated cultures, only approximately $35 \%$ of the $\mathrm{CTNT}^{+}$cells were also MLC-2V positive, with weak MLC-2V expression detected by western blots (Supplementary information, Figure S1). Collectively our results indicate that the majority of the cardiomyocytes in the Noggin+RAi-treated cultures are embryonic ventricular-like myocytes, while the cardiomyocytes differentiated in the Noggin+RAtreated cultures are either embryonic nodal- or atrial-like myocytes that do not express MLC-2V. We also examined the expression levels of $\beta$-MHC, MLC-2A, and atrial natriuretic factor (ANF) in RA- and RAi-treated cultures by western blotting, and the results showed that $\beta$-MHC is evenly expressed in the two cultures. How- ever, MLC-2A and ANF are expressed at higher levels in the Noggin+RA-treated cultures compared to the Noggin+RAi-treated cultures (Supplementary information, Figure S2).

Electrophysiological characterization identifies embryonic atrial- and ventricular-like cardiomyocyte populations induced by alternative retinoid signals

Due to a lack of endogenous early atrium-specific genetic markers in mammalian systems [22], we chose to use electrophysiological characteristics to rigorously identify these two cardiac sub-populations. Based on the morphology and classification of AP properties (Table 1) $[1,26]$, three major types of AP (nodal-like, atriallike, and ventricular-like) were observed in our study (Figure 4A). However, the ratios of the three major types of AP were different between the Noggin+RA- and Noggin+RAi-treated cultures. In the cultures treated with Noggin + RAi, $83 \%$ of myocytes $(n=23)$ possessed ventricular-like APs (Figure 4A and 4C), and the duration of the APs could be shortened by the application of the cal-

A
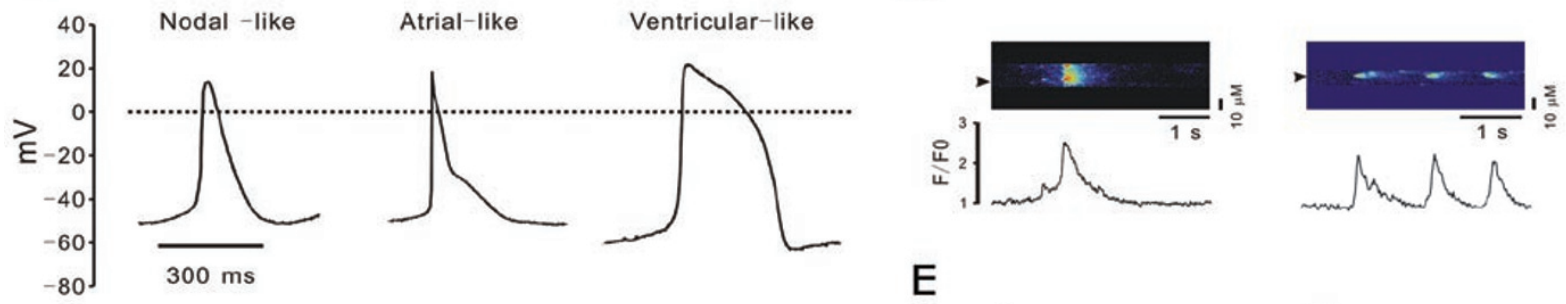

E
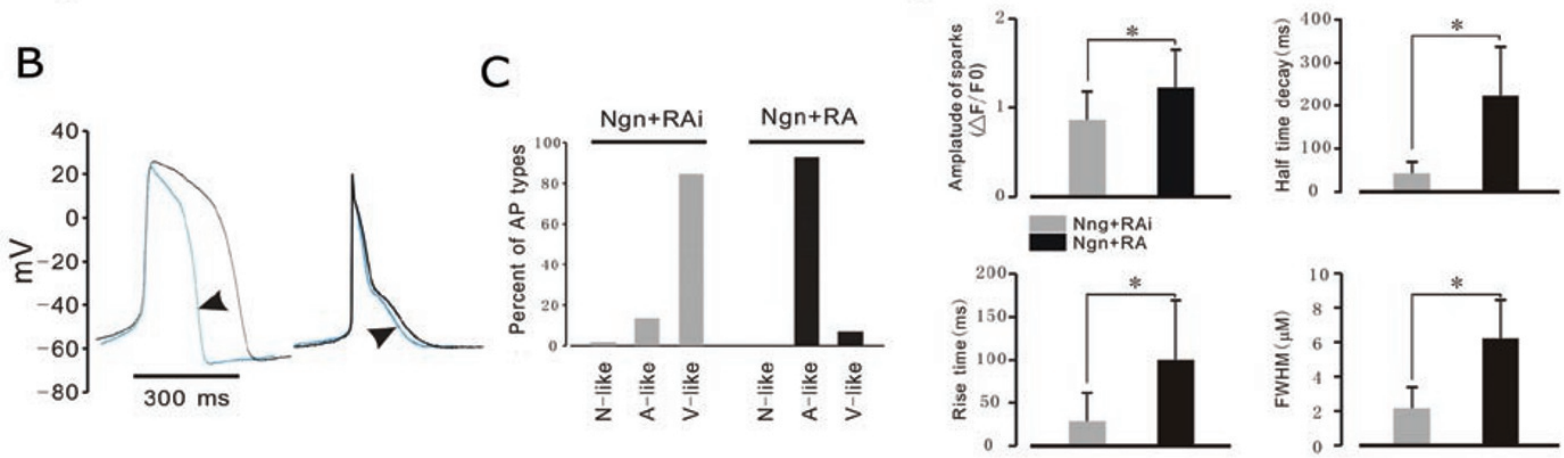

Figure $4 \mathrm{AP}$ morphologies and $\mathrm{Ca}^{2+}$ release properties of cardiomyocytes induced by $\mathrm{Ngn}+\mathrm{RA}$ and $\mathrm{Ngn}+\mathrm{RAi}$. (A) Three major types of APs were observed in hESC-derived cardiomyocytes: nodal-like, atrial-like, and ventricular-like. The duration of the ventricular-like APs was greatly reduced by the application of nifedipine (B, left); no significant effect of nifedipine on atrial-like AP duration was observed (B, right). (C) The percentages of the AP types recorded from Ngn+RA- and Ngn+RAtreated cultures are indicated. (D) Typical $\mathrm{Ca}^{2+}$ images recorded from cardiomyocytes in Ngn+RA cultures (left) and Ngn+RAi cultures (right). Fluorescence profiles (bottom) were taken from the images (at positions indicated by arrows). The properties of typical $\mathrm{Ca}^{2+}$-release events recorded from cardiomyocytes in Ngn+RA- and Ngn+RAi-treated cultures are summarized in (E) (98 sparks from 18 cells in Ngn+RA-treated cultures and 348 sparks from 14 cells in Ngn+RAi-treated cultures were measured). ${ }^{*} P<0.05$. All cells used in electrophysiological studies were 60 to 90 days old. $\mathrm{N}$-like, nodal-like AP; A-like, atrial-like AP; V-like, ventricular-like AP. 
Table 1 AP parameters recorded from hESC-derived cardiomyocytes

\begin{tabular}{lcclll}
\hline & $\mathrm{n}(\mathrm{cell})$ & $\mathrm{V} \max (\mathrm{V} / \mathrm{s})$ & $\mathrm{APA}(\mathrm{mV})$ & $\mathrm{APD} 90(\mathrm{~ms})$ & $\mathrm{MDP}(\mathrm{mV})$ \\
\hline Nodal-like & 6 & $7.3 \pm 4.2$ & $74.5 \pm 8.9^{\#}$ & $147.6 \pm 26.8^{\$}$ & $-51.3 \pm 8.2^{\dagger}$ \\
Atrial-like & 36 & $12.8 \pm 3.1^{*}$ & $81.6 \pm 11.5^{\#}$ & $168.8 \pm 26.8^{\$}$ & $-55.5 \pm 6.5^{\dagger}$ \\
Ventricular-like & 42 & $11.4 \pm 2.8^{*}$ & $86.8 \pm 12.4^{\#}$ & $285.8 \pm 52.6^{\$}$ & $-62.3 \pm 8.6^{\dagger}$ \\
\hline
\end{tabular}

Data are means \pm se. $n$ indicates the number of cells tested. Vmax, maximum rate of AP increase; APA. AP amplitude; APD90, AP duration measured at $90 \%$ repolarisation; MDP, maximum diastolic potential. ${ }^{*} P<0.05$ compared with nodal-like; ${ }^{\sharp} P<0.05$ compared with each other; ${ }^{\$} P<0.01$ compared with each other; and ${ }^{\dagger} P<0.05$ compared with each other.

cium channel blocker nifedipine (Figure 4B, left). However, $94 \%$ of the myocytes $(n=19)$ from Noggin+RAtreated cultures exhibited an atrial-like AP, and the duration of the AP could not be shortened by nifedipine (Figures $4 \mathrm{~A}, 4 \mathrm{~B}$ right and $4 \mathrm{C})$. These results demonstrate that the majority of the cardiomyocytes in the Noggin+RAtreated cultures were embryonic atrial-like myocytes, and the majority of those in the Noggin+RAi-treated cultures were embryonic ventricular-like myocytes. Interestingly, in both the Noggin+RA and Noggin+RAi-treated cultures, we did not observe the high percentages of cardiomyocytes bearing nodal-like APs that were reported in previous studies $[1,4]$.

There are important kinetic differences in $\mathrm{Ca}^{2+}$ sparks, the elementary unit of cardiomyocyte $\mathrm{Ca}^{2+}$ signaling, in atrial versus ventricular myocytes [27, 28]. $\mathrm{Ca}^{2+}$ sparks are significantly larger and longer lasting in atrial myocytes compared to ventricular myocytes [28, 29]. The results of imaging studies indicated that in the Noggin+RAi-treated culture, $87.5 \%(14 / 16)$ of the cells tested displayed $\mathrm{Ca}^{2+}$ sparks with a relatively low amplitude, fast rise time, short half time decay and small size. These are typical $\mathrm{Ca}^{2+}$ spark properties of ventricular-like myocytes (Figures 4D and 4E). On the other hand, in Noggin+RA-treated cultures, $81.8 \%$ (18/22) of the cells tested displayed $\mathrm{Ca}^{2+}$ sparks with a higher amplitude, slower rise time, longer half time decay and larger size (Figure 4D and 4E), suggesting that the myocytes from Noggin+RA-treated cultures were atrial-like myocytes. The kinetic study of $\mathrm{Ca}^{2+}$ release and the ratios of cardiomyocytes bearing those two patterns in the two cultures are consistent with and support previous AP phenotyping-based cardiac subtype categorization.

\section{Discussion}

Our results show that inhibition of BMP signaling after the initiation of cardiac differentiation promotes the cardiogenesis of hESCs. This finding is partially consistent with studies on mouse embryonic stem cells (mESCs) that demonstrate that the administration of Noggin before the initiation of differentiation promotes cardiogenesis [9]. Further comparing Noggin-treated cells with nontreated cells revealed that granulocyte colony-stimulating factor (G-CSF) promotes the proliferation of developing cardiomyocytes derived from mESCs [30]. Noggin sustains the undifferentiated proliferation of hESCs [31], and BMP4 is required for $\mathrm{mESC}$ self-renewal [32]. These different self-renewal mechanisms may be the cause of the differences observed in the cardiac differentiation studies of human and mouse embryonic stem cells.

Even though western blots revealed the differential expression of ANF and MLC-2A, both genes are expressed in 60-day-old Noggin+RA- and Noggin+RAi-treated cultures (Supplementary information, Figure S2). This result is consistent with Dr Rosenthal's statement, which indicated that there is no early atrial-specific marker in the mouse system. Instead, they used a proximal 840-bp quail SMyHC3 promoter to label the sino-atrial tissue from the earliest stage of heart development [22].

Previous studies of chicken and mouse embryos have proposed that RA signaling determines sinoatrial cell fate, whereas ventricular fate is specified in the absence of RA [17]. Our study shows that blocking RA signaling induces the expression of the ventricle-specific marker MLC-2V in major hESC-derived cardiomyocytes, and these cells possess APs and $\mathrm{Ca}^{2+}$ sparks typical of ventricular myocytes. Exogenous RA treatment directs the differentiation of hESCs into myocytes that retain the characteristic atrial-like APs and large $\mathrm{Ca}^{2+}$ sparks or $\mathrm{Ca}^{2+}$ transients. Our results demonstrate that the activation or inhibition of retinoid signals instructs the atrial versus ventricular specification of differentiating hESCs, respectively. By contrast, a previous study demonstrated that RA enhances the development of ventricular cardiomyocytes derived from mESCs [33]. This result could represent differences between the differentiation culture systems used in the two studies, the embryoid body procedure versus the flat culture system, or the timing of RA administration.

The potential risk of ventricular arrhythmias caused by the heterogeneity of hESC-derived cardiomyocytes 
is one of the major hurdles for the application of hESCs to cardiac repair $[1,5,6]$. The application of relatively homogeneous ventricular myocytes derived from hESCs in myocardial repair has a great potential to reduce this risk by removing one of the major barriers for developing hESC-based myocardial repair strategies. Recent advances in tissue engineering, including mouse ventricular progenitor cell isolation from $\mathrm{mESC}$ derivatives using a genetic labelling approach to generate functional ventricular muscles [34], suggest that human functional ventricular heart muscles can be generated with embryonic ventricular-like myocytes that are directly differentiated from hESCs. With a chemically defined culture system and no genetic manipulation, the direct differentiation procedures that we have developed could be easily employed in clinical studies of myocardial repair. Another challenge for developing hESC-based myocardial repair strategies is the development of biotechnology to rapidly generate the large amounts of ventricular myocytes required for transplantation. Our study demonstrated the efficient differentiation of embryonic atrial- and ventricular-like myocytes from hESCs by eliminating the embryoid body procedure, a time-consuming step commonly used in the cardiac differentiation of embryonic stem cells. If combined together with induced pluripotent stem (iPS) cell technology $[35,36]$, the programmed differentiation of atrial- and ventricular-like myocytes may not only be used to develop safe cell sources for personalised cardiac repair but also provide cellular models for the study of genetic atrial or ventricular diseases.

\section{Materials and Methods}

\section{Maintenance and differentiation of hESCS}

The undifferentiated hESC line H7 (WiCell Research Institute) was maintained on matrigel-coated plates, as previously described [37]. In the basic cardiac induction protocol (BP), undifferentiated hESCs were seeded on gelatin-coated plates at a density of $1-5 \times 10^{5} \mathrm{cells} / \mathrm{cm}^{2}$ and cultured with mouse embryonic fibroblastconditioned medium for 3 days until fully confluent. To initiate cell differentiation, the medium was changed to RPMI1640 and supplemented with B27 (Invitrogen). The cells were treated with $25 \mathrm{ng} / \mathrm{ml} \mathrm{BMP} 4$ and $6 \mathrm{ng} / \mathrm{ml} \mathrm{bFGF}$ at day $1100 \mathrm{ng} / \mathrm{ml}$ activin A at day 2, and $200 \mathrm{ng} / \mathrm{ml}$ DKK1 (R\&D Systems) from day 6 to day 11. The medium was changed every 3 days after day 11 (Figure 1). Noggin $(250 \mathrm{ng} / \mathrm{ml})$, RA (1 $\mu \mathrm{M}$, Sigma) or RAi $(1 \mu \mathrm{M})$ were added to the cell cultures at the times specified in Figures 1A-1C. Spontaneous beating clusters were typically observed on days 10 and 11. Cardiac differentiation efficiency was analysed on day 14 with CTNT antibody staining and flow cytometry analysis.

\section{Single-cell preparation of hESC-derived cardiomyocytes \\ Differentiated cultures that were 60 to 90 days old were washed in a low $\mathrm{Ca}^{2+}$ solution and then incubated in an enzyme solu- tion for $20 \mathrm{~min}$ at $37^{\circ} \mathrm{C}$. The dissociation was completed in $\mathrm{KB}$}

solution by gently shaking for $40 \mathrm{~min}$ at room temperature. The isolated cells were resuspended in DMEM plus $10 \%$ FBS, transferred to $0.1 \%$ gelatin-coated glass coverslips, and then kept in an incubator at $37{ }^{\circ} \mathrm{C}$ and $5 \% \mathrm{CO}_{2}$. The composition of the low$\mathrm{Ca}^{2+}$ solution was as follows: $120 \mathrm{mM} \mathrm{NaCl}, 5.4 \mathrm{mM} \mathrm{KCl}, 5 \mathrm{mM}$ $\mathrm{MgSO}_{4}, 5 \mathrm{mM}$ Na pyruvate, $20 \mathrm{mM}$ glucose, $20 \mathrm{mM}$ taurine, and $10 \mathrm{mM}$ HEPES. The $\mathrm{pH}$ was adjusted to 7.3 with $\mathrm{NaOH}$. The KB solution contained $85 \mathrm{mM} \mathrm{KCl}, 30 \mathrm{mM} \mathrm{K}_{2} \mathrm{HPO}_{4}, 5 \mathrm{mM} \mathrm{MgSO}_{4}$, $1 \mathrm{mM}$ EGTA, $2 \mathrm{mM} \mathrm{Na} \mathrm{ATP}_{2}, 5 \mathrm{mM}$ Na pyruvate, $20 \mathrm{mM}$ glucose, $20 \mathrm{mM}$ taurine, and $5 \mathrm{mM}$ creatine. The $\mathrm{pH}$ was adjusted to 7.3 with $\mathrm{KOH}$.

\section{Electrophysiological measurements and confocal $\mathrm{Ca}^{2+} \mathrm{im}-$ aging}

The APs of cardiomyocytes were recorded in a whole-cell patch clamp configuration using an Axon 200B amplifier (Axon Instruments) at room temperature. Data were digitised at $20 \mathrm{kHz}$, filtered at $2 \mathrm{kHz}$, and analysed by the PClamp 9.0 program. Patch pipettes (2-4 $\mathrm{M} \Omega$ resistance) were filled with an intracellular solution containing $50 \mathrm{mM} \mathrm{KCl}, 60 \mathrm{mM} \mathrm{K}$-aspartate, $1 \mathrm{mM} \mathrm{MgCl}_{2}$, $3 \mathrm{mM} \mathrm{Na}_{2} \mathrm{ATP}, 10 \mathrm{mM}$ EGTA, and $10 \mathrm{mM}$ HEPES. The $\mathrm{pH}$ was adjusted to 7.3 with $\mathrm{KOH}$. Normal Tyrode's solution was used as an extracellular solution and contained $140 \mathrm{mM} \mathrm{NaCl}, 5 \mathrm{mM} \mathrm{KCl}$, $1 \mathrm{mM} \mathrm{CaCl}_{2}, 1 \mathrm{mM} \mathrm{MgCl}_{2}, 10 \mathrm{mM}$ glucose, and $10 \mathrm{mM}$ HEPES. The $\mathrm{pH}$ was adjusted to 7.4 with $\mathrm{NaOH}$.

For $\mathrm{Ca}^{2+}$ confocal imaging, myocytes were incubated with Fluo-4AM (10 $\mu \mathrm{M} / \mathrm{L}$; Molecular Probes) for $10 \mathrm{~min}$ at room temperature and then perfused with extracellular buffer for approximately $30 \mathrm{~min}$. $\mathrm{Ca}^{2+}$ imaging studies were performed on a Leica SP5 confocal microscope equipped with an argon laser $(488 \mathrm{~nm})$ at a magnification of $40 \times$ using a 1.25 NA oil immersion objective. Spontaneous $\mathrm{Ca}^{2+}$ sparks and $\mathrm{Ca}^{2+}$ transients were recorded using linescans that were obtained at $0.5 \mathrm{~ms}$ per line. Images were processed and analysed using both MATLAB 7.1 software (MathWorks) and ImageJ (Scioncorp). Detection criteria of $3.8 \times \mathrm{SD}$ for $\mathrm{Ca}^{2+}$ sparks were set, and automated counting of $\mathrm{Ca}^{2+}$ sparks was performed using the Sparkmaster plug-in for ImageJ [38].

\section{Flow cytometry}

Differentiated cell clusters were dissociated into single cells with $0.25 \%$ trypsin-EDTA and were then fixed and stained with anti-human CTNT antibody (R\&D Systems) and goat anti-mouse FITC-conjugated secondary antibody (Santa Cruz) in PBS plus $0.5 \%$ BSA and $0.1 \%$ saponin (Sigma) at $4{ }^{\circ} \mathrm{C}$. Stained cells were kept in $4 \%$ paraformaldehyde for subsequent quantitative analysis. Data were collected using a FACScalibur (Becton Dickinson) and analysed with FlowJo software (Treestar).

\section{Real-time RT-PCR}

Total RNA was isolated from a single well of a 24-well plate of differentiated hESCs using Qiagen's RNeasy Plus Mini kit. Then, $1 \mu \mathrm{g}$ of total RNA was reverse transcribed with the SuperScript III First-Strand Synthesis System (Invitrogen). RT-PCR was performed using rTaq DNA Polymerase (Takara). Real-time PCR was performed in triplicate using the $2 \times$ QuantiFast SYBR Green I PCR Master Mix (Qiagen) on a Rotor Gene 6200 Real-Time PCR Machine (Corbett) with an annealing temperature of $60{ }^{\circ} \mathrm{C}$. The expression of each gene was normalized to GAPDH gene expression. Primer sequences are listed in Table 2. 
Table 2 Primer sequences used for qPCR

\begin{tabular}{llll}
\hline Gene & \multicolumn{1}{c}{ Forward Primer } & \multicolumn{1}{c}{ Reverse Primer } & Tm \\
\hline$N X K 2.5$ & acctcaacagctcctgactct & ataatcgccgccacaaactctcc & $60^{\circ} \mathrm{C}$ \\
$C T N T$ & ttcaccaaagatctgctcctcgct & ttattactggtgtggagtgggtgtgg & $60{ }^{\circ} \mathrm{C}$ \\
$I R X 4$ & ttccgttctgaagcgtggtc & tgaagcaggcaattattggtgt & $60{ }^{\circ} \mathrm{C}$ \\
GAPDH & gaaatcccatcaccatcttccagg & gagcccagccttctccatg & $60{ }^{\circ} \mathrm{C}$ \\
\hline
\end{tabular}

\section{Immunofluorescence}

Differentiated cultures (60 days old) were digested with $0.25 \%$ trypsin-EDTA, and the cells were plated on gelatin-coated coverslips for 5 days to allow full attachment to occur. Cells were then fixed in 4\% paraformaldehyde and incubated with primary antibodies: anti-human CTNT (R\&D systems), mouse anti-human $\alpha$-Actinin (Sigma), mouse anti-human $\beta$-MHC (ATCC), mouse anti-human MLC-2A (Synaptic Systems), or rabbit anti-human MLC-2V (ProteinTech Group). Goat anti-mouse secondary antibody conjugated with DyLight 488 (Santa Cruz Biotechnology) and goat anti-rabbit secondary antibody conjugated with Tritc (Santa Cruz Biotechnology) were used as needed. After the nuclei were counterstained with 4',6-diamidino-2-phenylindole (DAPI; Sigma), immunofluorescence images were visualised and recorded using an Olympus microscope system X51 or Olympus LSCM FV1000.

\section{Western blotting}

One well of a 24-well plate of 60-day-old differentiated cells was lysed with RIPA lysis buffer (Biomiga) for western blotting. Blots were incubated with mouse anti-human CTNT, mouse anti-human $\beta$-MHC, rabbit anti-human MLC-2V, goat anti-ANF, mouse anti-human MLC-2A, mouse anti-human $\beta$-actin, rabbit anti-phospho smad1/5/8 and rabbit anti-smad1/5/8 separately. Blots were then incubated with HRP-conjugated goat anti-mouse or antirabbit antibody.

\section{Acknowledgments}

We thank Drs Joy Fleming and Juanjuan Feng of the Institute of Biophysics, and Dr Paul Bornstein of the University of Washington for critical discussion and revision of the manuscript, and Dr Shigang He of the Institute of Biophysics for support and access to patch clamp technology. This work was supported by the Hi-Tech Research and Development Program of China (863 Program) (2006AA02A106), the National Basic Research Program of China (2006CB943901, 2010CB945024, and 2011CB965002), the Knowledge Innovation Program of the Chinese Academy of Sciences (KSCX2-YW-R-50), and the National Foundation of Science and Technology (30640005).

\section{References}

1 He JQ, Ma Y, Lee Y, Thomson JA, Kamp TJ. Human embryonic stem cells develop into multiple types of cardiac myocytes: action potential characterization. Circ Res 2003; 93:3239.

2 Laflamme MA, Chen KY, Naumova AV, et al. Cardiomyo- cytes derived from human embryonic stem cells in prosurvival factors enhance function of infarcted rat hearts. Nat Biotechnol 2007; 25:1015-1024.

3 Yang L, Soonpaa MH, Adler ED, et al. Human cardiovascular progenitor cells develop from a KDR+ embryonic-stem-cellderived population. Nature 2008; 453:524-528.

4 Zhu WZ, Xie Y, Moyes KW, et al. Neuregulin/ErbB signaling regulates cardiac subtype specification in differentiating human embryonic stem cells. Circ Res 2010; 107:776-786.

5 Kehat I, Khimovich L, Caspi O, et al. Electromechanical integration of cardiomyocytes derived from human embryonic stem cells. Nat Biotechnol 2004; 22:1282-1289.

6 Chen HS, Kim C, Mercola M. Electrophysiological challenges of cell-based myocardial repair. Circulation 2009; 120:2496-2508.

7 Tran TH, Wang X, Browne C, et al. Wnt3a-induced mesoderm formation and cardiomyogenesis in human embryonic stem cells. Stem Cells 2009; 27: 1869-1878.

8 Reppel M, Igelmund P, Egert U, et al. Effect of cardioactive drugs on action potential generation and propagation in embryonic stem cell-derived cardiomyocytes. Cell Physiol Biochem 2007; 19:213-224.

9 Yuasa S, Itabashi Y, Koshimizu U, et al. Transient inhibition of BMP signaling by Noggin induces cardiomyocyte differentiation of mouse embryonic stem cells. Nat Biotechnol 2005; 23:607-611.

10 Marvin MJ, Di Rocco G, Gardiner A, Bush SM, Lassar AB. Inhibition of Wnt activity induces heart formation from posterior mesoderm. Genes Dev 2001; 15:316-327.

11 Schneider VA, Mercola M. Wnt antagonism initiates cardiogenesis in Xenopus laevis. Genes Dev 2001; 15:304-315.

12 Korol O, Gupta RW, Mercola M. A novel activity of the Dickkopf-1 amino terminal domain promotes axial and heart development independently of canonical Wnt inhibition. Dev Biol 2008; 324:131-138.

$13 \mathrm{Xu}$ RH, Chen X, Li DS, et al. BMP4 initiates human embryonic stem cell differentiation to trophoblast. Nat Biotechnol 2002; 20:1261-1264.

14 Zhang P, Li J, Tan Z, et al. Short-term BMP-4 treatment initiates mesoderm induction in human embryonic stem cells. Blood 2008; 111:1933-1941.

15 Schulze GE, Clay RJ, Mezza LE, et al. BMS-189453, a novel retinoid receptor antagonist, is a potent testicular toxin. Toxicol Sci 2001; 59:297-308.

16 Keegan BR, Feldman JL, Begemann G, Ingham PW, Yelon D. Retinoic acid signaling restricts the cardiac progenitor pool. Science 2005; 307:247-249.

17 Xavier-Neto J, Rosenthal N, Silva FA, et al. Retinoid signaling and cardiac anteroposterior segmentation. Genesis 2001; 
31:97-104.

18 Patwardhan V, Fernandez S, Montgomery M, Litvin J. The rostro-caudal position of cardiac myocytes affect their fate. Dev Dyn 2000; 218:123-135.

19 Orts-Llorca F JCJ. Determination of heart polarity (arterio venous axis) in the chicken embryo. Roux Arch Entwicklungsmechanik 1967; 113:17.

20 Yutzey K, Gannon M, Bader D. Diversification of cardiomyogenic cell lineages in vitro. Dev Biol 1995; 170:531-541.

21 Hochgreb T, Linhares VL, Menezes DC, et al. A caudorostral wave of RALDH2 conveys anteroposterior information to the cardiac field. Development 2003; 130:5363-5374

22 Xavier-Neto J, Neville CM, Shapiro MD, et al. A retinoic acid-inducible transgenic marker of sino-atrial development in the mouse heart. Development 1999; 126:2677-2687.

23 Gassanov N, Er F, Zagidullin N, et al. Retinoid acid-induced effects on atrial and pacemaker cell differentiation and expression of cardiac ion channels. Differentiation 2008; 76:971980.

24 Bao ZZ, Bruneau BG, Seidman JG, Seidman CE, Cepko CL. Regulation of chamber-specific gene expression in the developing heart by Irx4. Science 1999; 283:1161-1164.

25 Fu JD, Jiang P, Rushing $\mathrm{S}$, et al. $\mathrm{Na}^{+} / \mathrm{Ca}^{2+}$ exchanger is a determinant of excitation-contraction coupling in human embryonic stem cell-derived ventricular cardiomyocytes. Stem Cells Dev 2010; 19:773-782.

26 Maltsev VA, Rohwedel J, Hescheler J, Wobus AM. Embryonic stem cells differentiate in vitro into cardiomyocytes representing sinusnodal, atrial and ventricular cell types. Mech Dev 1993; 44:41-50.

27 Cheng H, Lederer WJ. Calcium sparks. Physiol Rev 2008; 88:1491-1545.

28 Woo SH, Cleemann L, Morad M. Spatiotemporal characteristics of junctional and nonjunctional focal $\mathrm{Ca}^{2+}$ release in rat atrial myocytes. Circ Res 2003; 92:e1-e11.
29 Cleemann L, Wang W, Morad M. Two-dimensional confocal images of organization, density, and gating of focal $\mathrm{Ca} 2+$ release sites in rat cardiac myocytes. Proc Natl Acad Sci USA 1998; 95:10984-10989.

30 Shimoji K, Yuasa S, Onizuka T, et al. G-CSF promotes the proliferation of developing cardiomyocytes in vivo and in derivation from ESCs and iPSCs. Cell Stem Cell 2010; 6:227237.

$31 \mathrm{Xu}$ RH, Peck RM, Li DS, et al. Basic FGF and suppression of BMP signaling sustain undifferentiated proliferation of human ES cells. Nat Methods 2005; 2:185-190.

32 Ying QL, Nichols J, Chambers I, Smith A. BMP induction of Id proteins suppresses differentiation and sustains embryonic stem cell self-renewal in collaboration with STAT3. Cell 2003; 115:281-292.

33 Wobus AM, Kaomei G, Shan J, et al. Retinoic acid accelerates embryonic stem cell-derived cardiac differentiation and enhances development of ventricular cardiomyocytes. $J$ Mol Cell Cardiol 1997; 29:1525-1539.

34 Domian IJ, Chiravuri M, van der Meer P, et al. Generation of functional ventricular heart muscle from mouse ventricular progenitor cells. Science 2009; 326:426-429.

35 Takahashi K, Tanabe K, Ohnuki M, et al. Induction of pluripotent stem cells from adult human fibroblasts by defined factors. Cell 2007; 131:861-872.

36 Yu J, Vodyanik MA, Smuga-Otto K, et al. Induced pluripotent stem cell lines derived from human somatic cells. Science 2007; 318:1917-1920.

$37 \mathrm{Xu} \mathrm{C}$, Inokuma MS, Denham J, et al. Feeder-free growth of undifferentiated human embryonic stem cells. Nat Biotech 2001; 19:971-974

38 Picht E, Zima AV, Blatter LA, Bers DM. SparkMaster: automated calcium spark analysis with ImageJ. Am J Physiol Cell Physiol 2007; 293:C1073-C1081.

(Supplementary information is linked to the online version of the paper on the Cell Research website.) 